\title{
大陆俯冲带流体活动和元素迁移: 大别-苏鲁超高压 变质岩和变质脉的研究
}

\author{
黄建, 肖益林* \\ 中国科学院壳幔物质与环境重点实验室, 中国科学技术大学地球和空间科学学院, 合肥 230026 \\ * 联系人, E-mail: ylxiao@ustc.edu.cn \\ 2013-06-05 收稿, 2013-06-18 接受, 2013-07-29 网络版发表 \\ 国家重点基础研究发展计划(2009CB825002)和国家自然科学基金(41273037, 41090372)资助
}

\begin{abstract}
摘要 大别-苏鲁超高压变质岩和变质脉的岩石学及地球化学研究表明, 在大陆俯冲带流体-岩石 反应过程中，伴随着显著的元素迁移和同位素分馏。大别-苏鲁造山带高压脉中单矿物(如金红石 和多硅白云母)具有明显的生长环带, 指示变质脉是流体-岩石相互作用的结果. 较高的流体/岩石 比和强烈的二者相互作用是导致 $\mathrm{Nb}-\mathrm{Ta}$ 迁移和分异的主导因素. 对中国大陆科学钻探主孔石榴 橄榄岩、榴辉岩和变质脉的 $\mathrm{Li}$ 和 $\mathrm{B}$ 同位素地球化学研究, 表明在陆壳深俯冲和折返过程中 $\mathrm{Li}$ 和 $\mathrm{B}$ 发生了明显的分馏且表现出不同的地球化学行为. 富集 $\mathrm{Si}, \mathrm{Al}, \mathrm{Na}, \mathrm{LILE}$ (如 Rb, Ba 和 $\mathrm{Sr}$ ) 和 $\operatorname{HFSE}$ (如 $\mathrm{Nb}, \mathrm{Ta}$ 和 $\mathrm{Ti}$ ) 的高压变质脉的存在以及不同岩性接触带高度变化的地球化学特征指示, 在峰期超高压变质条件下可能形成了超临界流体, 它对微量元素的迁移起到了非常重要的作用.
\end{abstract}

关键词 大陆深俯冲 流体活动 变质脉 元素迁移 同位素分馏
俯冲带的变质流体不仅对岛弧岩浆岩的形成和 地幔不均一性的产生具有重要意义, 而且能够影响 俯冲带变质过程中元素和同位素的地球化学行为 ${ }^{[1,2]}$. 在过去的 40 年里, 对洋壳俯冲变质过程中变质流体 的来源、性质、运移方式以及作用都有了较为详细的 研究 ${ }^{[2,3]}$, 然而对大陆碰撞过程中变质流体的研究还 较为缺乏 ${ }^{[4]}$. 大别-苏鲁造山带是目前世界上已发现 的规模最大、出露最好的超高压变质地体之一, 它是 由华南板块俯冲进人华北板块之下所形成的三叠纪 陆-陆碰撞造山带, 造山带内含有大量的超高压变质 岩(如榴辉岩、片麻岩、橄榄岩、硬玉石英岩和大理 岩等) 和变质脉 ${ }^{[5]}$. 其中, 变质脉进一步可以细分为 石英脉 (石英体积分数 $>98 \%$ ) 和复杂矿物组合脉. 详 细研究这些变质脉不仅能够揭示成脉流体的来源、组 成和演化, 为大陆俯冲带流体活动提供新的制约, 而 且能够制约大陆俯冲带元素和同位素的迁移和分异 行为 ${ }^{[6 \sim 10]}$. 本文以大别造山带典型地区 (碧溪岭和朱
家冲)和中国大陆科学钻探主孔的超高压变质岩和变 质脉为研究对象, 结合详细的岩相学和精确的地球 化学数据 (主微量元素和 Sr-Nd-Pb-Li-B-O 同位素), 试图揭示大陆俯冲带流体活动、元素(如 $\mathrm{Nb}, \mathrm{Ta}$ )迁移 和同位素(如 $\mathrm{Li}, \mathrm{B}$ )分馏的内在联系.

\section{1 中大别碧溪岭超高压榴辉岩和变质脉的 研究}

野外关系、岩石学和地球化学证据都表明, 大别 造山带中部的碧溪岭镁铁-超镁铁质岩体的原岩是源 自地幔的玄武质岩浆堆积形成的堆积岩 ${ }^{[11,12]}$. 矿物 氧同位素、流体包裹体和名义上无水矿物的结构水含 量研究表明, 在整个俯冲和折返构造循环中流体活 动非常有限 ${ }^{[13 ~ 16]}$. 因此, 直接比较碧溪岭远离脉榴 辉岩和没有经历变质的火成岩原岩一扬子新元古 代辉长岩的地球化学特征, 能够定性地评估俯冲带水岩相互作用较弱时, $\mathrm{Nb}-\mathrm{Ta}$ 的地球化学行为. 另外,

引用格式: 黄建, 肖益林. 大陆俯冲带流体活动和元素迁移: 大别-苏鲁超高压变质岩和变质脉的研究. 科学通报, 2013, 58: 2180-2185 Huang J, Xiao Y L. Fluid flow and element mobility in continental subduction zones: A study of UHP metamorphic rocks and veins in the Dabie-Sulu orogenic belt. Chin Sci Bull, 2013, 58: 2180-2185, doi: 10.1360/972013-600 
对比靠近脉榴辉岩和脉的地球化学特征, 可以制约强 烈水-岩相互作用过程中, $\mathrm{Nb}-\mathrm{Ta}$ 的迁移和分异行为.

我们的分析结果显示(图 1(a)), 远离脉榴辉岩的 $\mathrm{Nb}$ 和 $\mathrm{Ta}$ 含量分别为 $0.42 \sim 3.97$ 和 $0.03 \sim 0.24 \mathrm{ppm}$ $\left(1 \mathrm{ppm}=1 \mu \mathrm{g} \mathrm{g}^{-1}\right.$, 余同), $\mathrm{Nb} / \mathrm{Ta}$ 比值为 $14.0 \sim 19.2$, 落在 扬子辉长岩的 $\mathrm{Nb} / \mathrm{Ta}$ 比值范围之内 $(12 \sim 21)$. 同时, 两 者具有相似的平均 $\mathrm{Nb} / \mathrm{Ta}$ 比值, 分别为 $16.9 \pm 0.8$ (2 s.e. $n=12)$ 和 $16.2 \pm 0.6(2$ s.e. $n=78)$. 根据 $1 g-1 g$ 微量元素含 量判别图解(分配系数相同的 2 个元素会构成一条斜 率为 1 的直线), 我们以远离脉榴辉岩的 $\mathrm{Nb}-\mathrm{Ta}$ 含量, 做 $\lg [\mathrm{Nb}]$ 对 $\lg [\mathrm{Ta}]$ 图解, 获得了一条斜率为 $0.965 \pm$ 0.038 的直线. 这些结果说明, 在水/岩相互作用较弱 时, 由镁铁质岩石 (如辉长岩和玄武岩)进变质转变为 榴辉岩的过程中, $\mathrm{Nb}-\mathrm{Ta}$ 几乎不发生迁移和分异 ${ }^{[9]}$.

相反, 靠近脉榴辉岩的 $\mathrm{Nb} / \mathrm{Ta}$ 比值相对较高, 远 高于对应的变质脉(图 1(b)), 说明局部地区强烈的水岩相互作用能导致显著的 $\mathrm{Nb}-\mathrm{Ta}$ 迁移和分异. 此外, 脉金红石表现显著的核部低边部高的 $\mathrm{Nb} / \mathrm{Ta}$ 环带，与
靠近脉榴辉岩中金红石的 $\mathrm{Nb} / \mathrm{Ta}$ 环带互补(图 1(c), (d)), 说明成脉流体来自围岩的脱水. 脉金红石存在 显著的 $\mathrm{Nb} / \mathrm{Ta}$ 环带，暗示它是不同的变质阶段多期次 结晶的结果, 也说明成脉流体的 $\mathrm{Nb} / \mathrm{Ta}$ 比值由早期的 低值演化到晚期的高值. 在金红石出现之前, 角闪石 控制着流体中 $\mathrm{Nb}-\mathrm{Ta}$ 的分配 ${ }^{[18]}$ 且低 $\mathrm{Mg} \#(<75)$ 角闪石 倾向于富集 $\mathrm{Nb}^{[19]}$, 故早期角闪岩脱水会产生低 $\mathrm{Nb} / \mathrm{Ta}$ 的变质流体, 它们至少有部分被储存在岩石的 裂隙或空隙内, 与岩石一起俯冲到深部. 当变质压力 高于玄武岩 $-\mathrm{H}_{2} \mathrm{O}$ 体系的第二临界点压力时, 富水流 体转化为具有较高 $\mathrm{Nb} / \mathrm{Ta}$ 的超临界流体 ${ }^{[20]}$. 脉金红 石核部从早期的富水流体中结晶, 表现较低的 $\mathrm{Nb} / \mathrm{Ta}$ 比值; 而边部从晚期的超临界流体中结晶, 具有较高 的 $\mathrm{Nb} / \mathrm{Ta}$ 比值(图 1(c)).

\section{2 中国大陆科学钻探主孔超高压变质岩和 变质脉的研究}

为了进一步制约大陆俯冲和折返过程中的流体
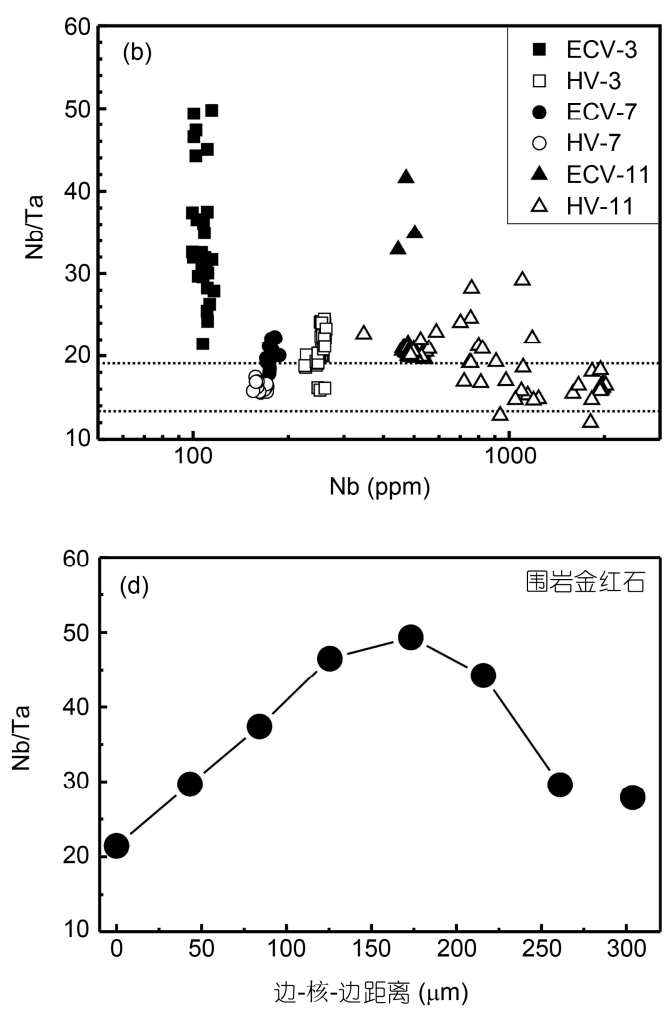

图 1 大别山中部碧溪岭超高压榴辉岩和变质脉中金红石的 $\mathrm{Nb} / \mathrm{Ta}$ 比值 ${ }^{[9]}$

(a) 远离脉榴辉岩(EAV)的 $\mathrm{Nb}$ 含量和 $\mathrm{Nb} / \mathrm{Ta}$ 图解; (b) 靠近脉榴辉岩和脉金红石的 $\mathrm{Nb} / \mathrm{Ta}$ 对比图解. 榴辉岩 ECV-3, 7, 11 分别对应脉 HV-3, 7, 11; (c) 脉金红石边-核-边 $\mathrm{Nb} / \mathrm{Ta}$ 剖面; (d) 靠近脉榴辉岩中金红石边-核-边 $\mathrm{Nb} / \mathrm{Ta}$ 剖面. 大洋中脊玄武岩(MORB)和洋岛玄武岩(OIB) 区域据 Münker 等人 ${ }^{[17]}$ 同位素稀释法所测结果; 上、下虚线分别表示球粒陨石和 $\mathrm{MORB}$ 的平均 $\mathrm{Nb} / \mathrm{Ta}$ 比值 ${ }^{[17]}$ 
活动、元素迁移、同位素分馏和壳-幔相互作用, 我们 从中国大陆科学钻探主孔仔细挑选了 4 组样品进行研 究 ${ }^{[7]}$. 第 1 组样品包括榴辉岩和高压变质脉, 脉中含 有高压矿物组合多硅白云母+石英+绿辉石; 第 2 组样品 由地幔楔石榴橄榄岩、榴辉岩和两者之间的过渡带组 成; 第 3 组样品由新鲜榴辉岩和退变质的角闪岩组成; 第 4 组样品包括新鲜榴辉岩和退变质形成的角闪岩脉.

\section{1 进变质过程}

榴辉岩和高压变质脉能够用来讨论陆壳俯冲脱 水过程中元素和同位素的地球化学行为, 原因在于: (1) 榴辉岩和脉中石榴石、绿辉石和多硅白云母具有 相似的氧同位素组成, 说明成脉流体为内生流体, 即 由直接围岩脱水产生; (2) 脉多硅白云母显示核部低 ( 3.3 pfu Si)、幔部高( 3.6 pfu Si) 和边部低 $\mathrm{Si}(\sim 3.3 \mathrm{pfu}$ $\mathrm{Si}$ )的成分环带, 对应的变质压力分别为 $2.5, \sim 3.5$ (与 榴辉岩的峰期变质压力一致) 和 $2.5 \mathrm{GPa}^{[7]}$, 说明脉 与榴辉岩一起经历了进、峰期和退变质作用. 与榴辉 岩相比, 脉石榴石和绿辉石以及脉全岩含有较高的 LILE(如 $\mathrm{Li}, \mathrm{Rb}, \mathrm{Sr}$ 和 Ba), LREE 和 HFSE(如 Ti, Nb 和 $\mathrm{Ta}$ )含量. 由于富水流体中溶质含量较低, 不能迁 移大量的微量元素 ${ }^{[21,22]}$, 因此早期含水矿物 (如硬柱 石、角闪石和钠云母)脱水产生的富水流体在超高压 变质阶段可能转变为溶解能力极强的超临界流体, 从而导致微量元素由榴辉岩短距离迁移至变质脉 中 $^{[7,10,23]}$.

与轻同位素 ${ }^{6} \mathrm{Li}$ 和 ${ }^{10} \mathrm{~B}$ 相比, 重同位素 ${ }^{7} \mathrm{Li}$ 和 ${ }^{11} \mathrm{~B}$ 更易于进人流体相 ${ }^{[24]}$, 因此板片俯冲脱水产生的流 体应该具有比残留榴辉岩更重的 $\mathrm{Li}$ 和 B 同位素组成. 但是在本研究中(图 2), 脉的 $\mathrm{Li}$ 同位素组成(1.2\%) 比 榴辉岩 $(-1.6 \%)$ 偏重, 而 B 同位素组成偏轻 $(0.9 \%$ 。和 $3.1 \%$ ), 说明在变质脱水过程中 $\mathrm{Li}$ 和 B 表现出不同的 地球化学行为. 这进一步指示, 高压/超高压变质岩 中的变质脉可能不是直接从俯冲板片脱水产生的初 始流体中沉淀的, 而是经历了流体-岩石相互作用之 后沉淀的产物 ${ }^{[9]}$.

\section{2 壳一慢相互作用}

地壳和地幔之间的物质和能量交换一直是地球 科学研究的热点问题. 本研究所选取的石榴橄榄岩 和榴辉岩之间的过渡带可以看作是壳-幔相互作用的 直接记录. 与石榴橄榄岩相比, 过渡带表现的显著特
征如下 ${ }^{[7]}$ : (1) 含有新生成的角闪石和绿泥石; (2) 具 有较高的 $\mathrm{Rb}, \mathrm{Ba}, \mathrm{Sr}$ 和 LREE 元素含量; (3) B 和 O 同 位素组成分别为 $5.8 \%$ 和 $3.3 \%$, 介于石榴橄榄岩 $(11.7 \% \circ$ 和 $3.9 \%$ ) 和榴辉岩 $(-0.1 \% \circ$ 和 $3.1 \% \circ)$ 之间. 这些 观察指示, 在大陆俯冲带, 地幔橄榄岩受到了来自深 俯冲陆壳的富含硅酸盐组分的流体的交代. 石榴橄 榄岩含有 $7.5 \mathrm{ppm}$ 的 $\mathrm{Li}$ 和 $4.5 \mathrm{ppm}$ 的 $\mathrm{B}$, 明显高于典 型地幔的 $\mathrm{Li}(<2 \mathrm{ppm})$ 和 $\mathrm{B}(<1 \mathrm{ppm})$ 含量 ${ }^{[25]}$, 说明壳源 $\mathrm{Li}$ 和 B 的加人对地幔的 $\mathrm{Li}$ 和 B 含量具有重要的影响. 石榴橄榄岩、榴辉岩和过渡带三者之间具有较小的 Li 同位素分馏(1.8\% 2.5\%), 但是却表现较大的 B 同 位素分馏(-0.1\% 11.7\% ), 说明壳-幔相互作用过程 中 $\mathrm{Li}$ 和 $\mathrm{B}$ 表现为不同的地球化学行为.

\section{3 退变质过程}

形成退变质角闪岩脉的流体主要来自围岩榴辉 岩的脱水作用, 证据在于: (1) 两者具有相似的 ${ }^{87} \mathrm{Sr} /$ ${ }^{86} \mathrm{Sr}$ 组成 $(\sim 0.70630)$; (2) 石榴石具有相同的 O 同位素 组成 $(1.8 \%$ ); (3) 两者含有相似的主微量元素含量 (HREE 除外, 榴辉岩含有稍高的 HRRE 含量). 在深 俯冲陆壳折返过程中, 含水矿物的分解以及分子水 和结构差基的出溶是形成角闪岩脉的退变质流体的 主要来源 ${ }^{[4]}$. 另外, 榴辉岩和角闪岩脉具有相同的 B 含量 $(0.4 \mathrm{ppm})$ 和 B 同位素 $(-5.0 \%$ )组成, 但是前者比 后者含有较高的 $\mathrm{Li}$ 含量 $(18$ 和 $7.7 \mathrm{ppm}$ ) 和较低的 $\mathrm{Li}$ 同位素(-6.9\% 和 $-0.4 \%$ )组成(图 2). 这个差别说明,

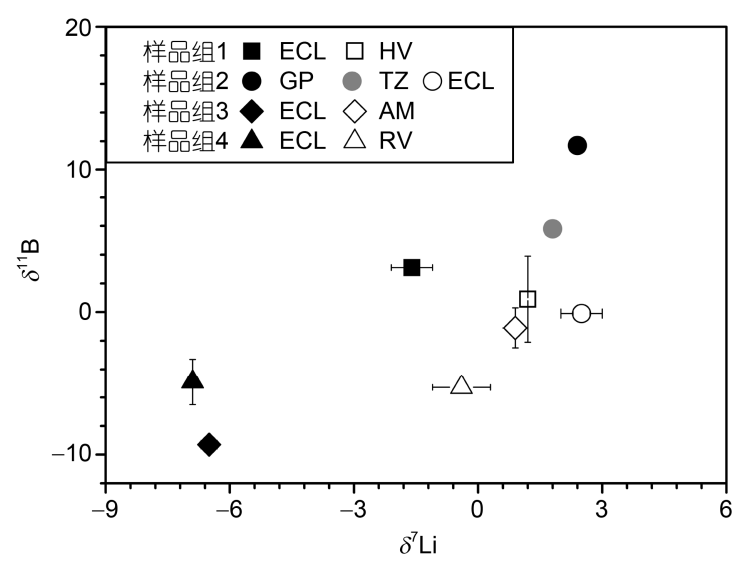

图 2 中国大陆科学钻探主孔超高压变质岩和变质脉的 $\delta^{7} \mathbf{L i}$ 和 $\delta^{11} \mathrm{~B}$ 同位素组成 ${ }^{[7]}$

样品组 1 和 2 分别包括榴辉岩(ECL)-高压变质脉(HV)和石榴橄榄岩 (GP)-过渡带(TZ)-榴辉岩; 样品组 3 和 4 分别包括榴辉岩-退变质角闪 岩(AM) 和榴辉岩-角闪岩脉(RV) 
退变质过程中内生流体不会导致 B 同位素的明显分 馏, 而榴辉岩和角闪岩脉在矿物组合上的差别可能 是导致两者 $\mathrm{Li}$ 含量和 $\mathrm{Li}$ 同位素组成不同的主要原 因 ${ }^{[7]}$. 另外, 退变质角闪岩的 $\mathrm{O}, \mathrm{Li}$ 和 $\mathrm{B}$ 同位素以及 ${ }^{87} \mathrm{Sr} /{ }^{86} \mathrm{Sr}$ 组成分别为 $2.52 \%, 0.9 \%,-1.1 \%$ 和 0.71123 , 明显高于对应的新鲜榴辉岩 $(1.21 \%,-6.5 \%,-9.3 \%$ 。 和 0.70633), 说明有外来流体参与了退变质角闪岩的 形成 ${ }^{[7]}$.

\section{3 南大别朱家冲榴辉岩和花岗片麻岩过渡 带的研究}

俯冲带存在 3 种不同类型的流体, 即富水流体、 含水熔体和超临界流体 ${ }^{[21,22]}$. 虽然实验研究显示俯 冲带变质条件下超临界流体能够出现 ${ }^{[20,26]}$, 但是在 天然超高压岩石样品中识别超临界流体活动的地球 化学证据尚属难题. 不过, 前人在研究超高压变质岩 的微量元素地球化学时, 已经推测超临界流体对微 量元素迁移的作用 ${ }^{[10,23,27]}$. 为了揭示大陆俯冲过程中 可能存在的超临界流体, 我们研究了大别造山带南 部朱家冲地区角闪岩(榴辉岩退变质的产物)和花岗
片麻岩过渡带的元素和同位素地球化学. 这个变质 带的岩石经过了低温/超高压变质作用 ${ }^{[28]}$, 对花岗片 麻岩中锆石的研究已经发现了超临界流体作用的地 球化学记录 ${ }^{[27]}$. 我们的主要研究结果如下: (1) 岩相 学研究显示, 在靠近接触带的角闪岩的石榴石中, 存 在由钾长石 + 石英 + 方解石 + 锆石 + 石榴石/角闪石/斜 䁫穷石组成的多相固体包裹体; (2) 主微量元素分析 显示(图 3), 相比其他角闪岩, 靠近接触带的角闪岩 具有较高的 K, Al, LILE, REE 和 HFSE 含量以及相对 较低的 $\mathrm{Mg}-\mathrm{Ca}$ 含量, 但它们的 $\mathrm{SiO}_{2}$ 含量接近; (3) 全 岩 $\mathrm{O}$ 同位素分析显示, 靠近接触带的片麻岩的 $\delta^{18} \mathrm{O}$ 值略有降低而角闪岩的 $\delta^{18} \mathrm{O}$ 值逐渐升高, 说明接触 带的流体活动导致了角闪岩和花岗片麻之间的 $\mathrm{O}$ 同 位素交换.

实验岩石学结果表明, 花岗岩 $-\mathrm{H}_{2} \mathrm{O}$ 体系的第二 临界点位于 2.5 3.0 GPa/700 ${ }^{\circ} \mathrm{C}^{[21,22]}$, 而玄武岩 $-\mathrm{H}_{2} \mathrm{O}$ 体系的第二临界点位于 3.4 5.5 GPa/800 1000 ${ }^{\circ} \mathrm{C}^{[20,26]}$. 结合南大别超高压变质岩的 $P-T$ 轨迹和峰期超高压 变质条件 $\left(\sim 3.3 \mathrm{GPa} / 670^{\circ} \mathrm{C}\right)^{[28]}$ 和折返 $P-T-t$ 轨迹 ${ }^{[29]}$ 以及玄 武岩/花岗岩 $-\mathrm{H}_{2} \mathrm{O}$ 体系的相图, 理论上超临界流体
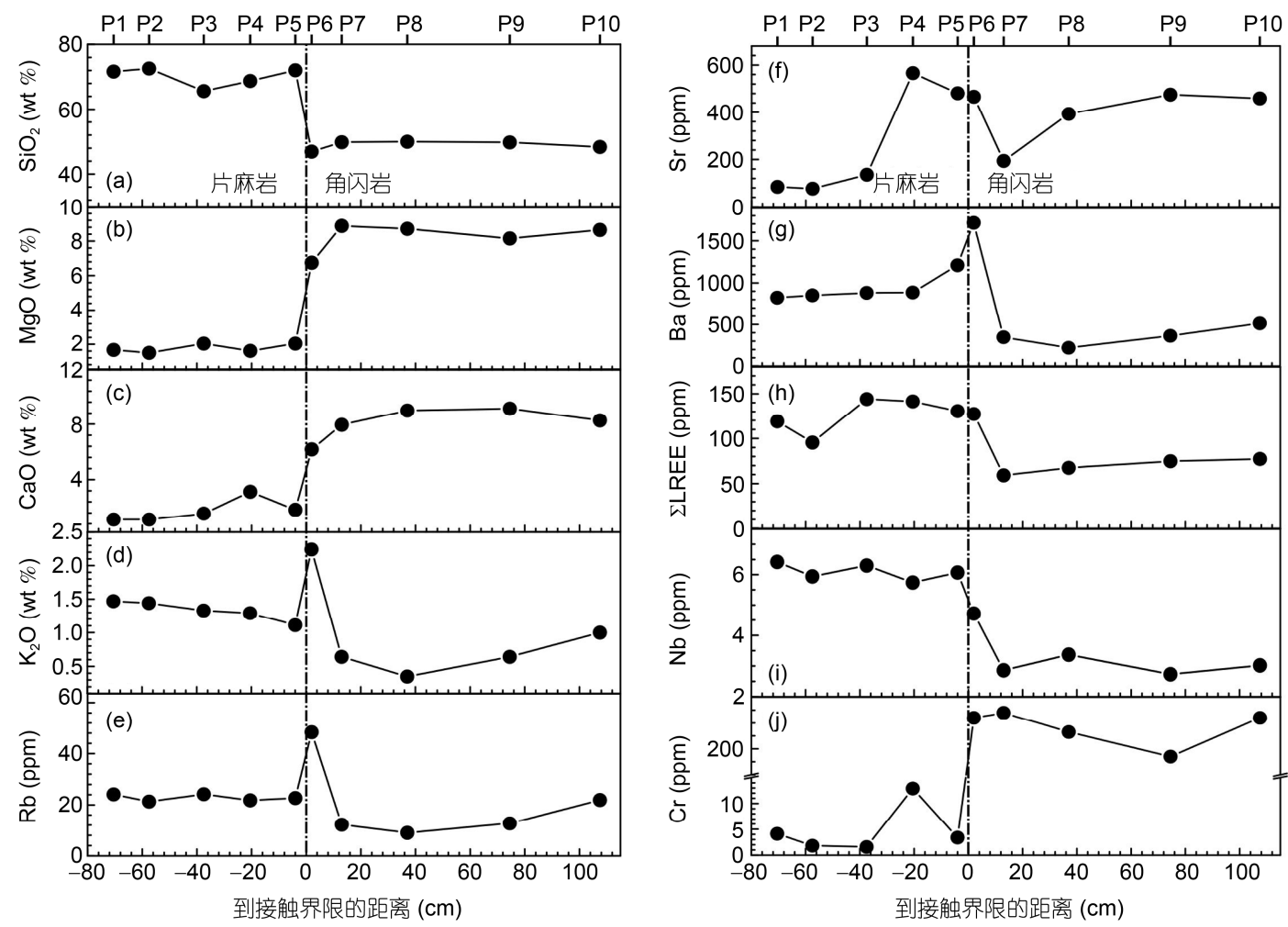

图 3 沿着角闪岩和花岗片麻岩剖面的主微量元素变化 
可以在峰期超高压变质条件下的花岗片麻岩中形成. 在折返过程中随着压力的降低发生相分离形成含水 熔体和富水流体 ${ }^{[22,27]}$, 从而形成石榴石中的多相固 体包裹体 ${ }^{[30]}$. 角闪岩相退变质作用和富水流体交代 对岩石的 HREE 和 HFSE 没有影响, 含水熔体的交代 则会产生 $\mathrm{SiO}_{2}$ 的富集, 这些都与本研究的数据结果 不符. 而超临界流体的组成变化很大, 可以从类似富
水流体的组成变化到类似含水熔体的组成 ${ }^{[21,31]}$, 但 却具有极强的溶解能力 ${ }^{[20,22]}$. 超临界流体致使片麻 岩中富集 HFSE 和 HREE 元素的副矿物(如金红石、 石榴石和褐帘石)分解。在峰期变质条件下，这种富 含 LILE, HFSE 和 REE 的流体汇聚在不同岩性接触带 并交代紧邻的榴辉岩, 导致岩性接触带边界角闪岩 样品主微量元素含量发生显著的变化(图 3).

\section{参考文献}

1 Zheng Y F. Metamorphic chemical geodynamics in continental subduction zones. Chem Geol, 2012, 328: 5-48

2 Spandler C, Pirard C. Element recycling from subducting slabs to arc crust: A review. Lithos, 2013, 170-171: 208-223

3 Bebout G E. Metamorphic chemical geodynamics of subduction zones. Earth Planet Sci Lett, 2007, 260: 373-393

4 Zheng Y F. Fluid regime in continental subduction zones: Petrological insights from ultrahigh-pressure metamorphic rocks. J Geol Soc Lond, 2009, 166: 763-782

5 郑永飞. 超高压变质与大陆碰撞研究进展：以大别-苏鲁造山带为例. 科学通报, 2008, 53: 2129-2152

6 Zheng Y F, Gao T S, Wu Y B, et al. Fluid flow during exhumation of deeply subducted continental crust: Zircon U-Pb age and O isotope studies of quartz vein in eclogite. J Metamorph Geol, 2007, 25: 267-283

7 Xiao Y L, Hoefs J, Hou Z H, et al. Fluid/rock interaction and mass transfer in continental subduction zones: Constraints from trace elements and isotopes ( $\mathrm{Li}, \mathrm{B}, \mathrm{O}, \mathrm{Sr}, \mathrm{Nd}, \mathrm{Pb}$ ) in UHP rocks from the Chinese Continental Scientific Drilling Program, Sulu, East China. Contrib Mineral Petrol, 2011, 162: 797-819

8 Guo S, Ye K, Chen Y, et al. Fluid-rock interaction and element mobilization in UHP metabasalt: Constraints from an omphacite-epidote vein and host eclogites in the Dabie orogen. Lithos, 2012, 136-139: 145-167

9 Huang J, Xiao Y L, Gao Y J, et al. Nb-Ta fractionation induced by fluid-rock interaction insubduction-zones: Constraints from UHP eclogite- and vein-hosted rutile from the Dabieorogen, Central-Eastern China. J Metamorph Geol, 2012, 30: 821-842

10 Sheng Y M, Zheng Y F, Li S N, et al. Element mobility during continental collision: Insights from polymineralic metamorphic vein within UHP eclogite in the Dabie orogen. J Metamorph Geol, 2013, 31: 221-241

11 Zhang R Y, Liou J G, Cong B L. Talc-, magnesite- and Ti-clinohumite-bearing ultrahigh-pressure meta-mafic and ultramafic complex in the Dabie Mountains, China. J Petrol, 1995, 36: 1011-1037

12 Tang H F, Liu C Q, Nakai S I, et al. Geochemistry of eclogites from the Dabie-Sulu terrane, eastern China: New insights into protoliths and trace element behavior during UHP metamorphism. Lithos, 2007, 95: 441-457

13 Zheng Y F, Fu B, Xiao Y L, et al. Hydrogen and oxygen isotope evidence for fluid-rock interactions in the stages of pre- and post-UHP metamorphism in the Dabie Mountains. Lithos, 1999, 46: 677-693

14 Xiao Y L, Hoefs J, van den Kerkhof A M, et al. Fluid history of UHP metamorphism in Dabie Shan, China: A fluid inclusion and oxygen isotope study on the coesite-bearing eclogite from Bixiling. Contrib Mineral Petrol, 2000, 139: 1-16

15 Xia Q K, Sheng Y M, Yang X Z, et al. Heterogeneity of water in garnets from UHP eclogites, eastern Dabieshan, China. Chem Geol, 2005, 224: $237-246$

16 Sheng Y M, Xia Q K, Dallai L, et al. $\mathrm{H}_{2} \mathrm{O}$ contents and D/H ratios of nominally anhydrous minerals from ultrahigh-pressure eclogites of the Dabie orogen, eastern China. Geochim Cosmochim Acta, 2007, 71: 2079-2103

17 Münker C, Pfänder J A, Weyer S, et al. Evolution of planetary cores and the earth-moon system from Nb/Ta systematics. Science, 2003, 301: $84-87$

18 Xiao Y L, Sun W D, Hoefs J, et al. Making continental crust through slab melting: Constraints from niobium-tantalum fractionation in UHP metamorphic rutile. Geochim Cosmochim Acta, 2006, 70: 4770-4782

19 Tiepolo M, Vannucci R, Oberti R, et al. Nb and Ta incorporation and fractionation in titanian pargasite and kaersutite: Crystal-chemical constraints and implications for natural systems. Earth Planet Sci Lett, 2000, 176: 185-201

20 Kessel R, Schmidt M W, Ulmer P, et al. Trace element signature of subduction-zone fluids, melts and supercritical liquids at $120-180 \mathrm{~km}$ depth. Nature, 2005, 437: 724-727 
21 Hermann J, Spandler C, Hack A, et al. Aqueous fluids and hydrous melts in high-pressure and ultra-high pressure rocks: Implications for element transfer in subduction zones. Lithos, 2006, 92: 399-417

22 Zheng Y F, Xia Q X, Chen R X, et al. Partial melting, fluid supercriticality and element mobility in ultrahighpressure metamorphic rocks during continental collision. Earth-Sci Rev, 2011, 107: 342-374

23 Zhang Z M, Shen K, Sun W D, et al. Fluids in deeply subducted continental crust: Petrology, mineral chemistry and fluid inclusion of UHP metamorphic veins from the Sulu orogen, eastern China. Geochim Cosmochim Acta, 2008, 72: 3200-3228

24 Moriguti T, Shibata T, Nakamura E. Lithium, boron and lead isotope and trace element systematics of Quaternary basaltic volcanic rocks in northeastern Japan: Mineralogical controls on slab-derived fluid composition. Chem Geol, 2004, 212: 81-100

25 Paquin J, Altherr R, Ludwig T. Li-Be-B systematics in the ultrahigh-pressure garnet peridotite from AlpeArami (Central Swiss Alps): Implications for slab-to-mantle wedge transfer. Earth Planet Sci Lett, 2004, 218: 507-519

26 Mibe K, Kawamoto T, Matsukage K N, et al. Slab melting versus slab dehydration in subduction-zone magmatism. Proc Natl Acad Sci USA, 2011, 108: 8177-8182

27 Xia Q X, Zheng Y F, Hu Z C. Trace elements in zircon and coexisting minerals from low-T/UHP metagranite in the Dabie orogen: Implications for action of supercritical fluid duringcontinental subduction-zone metamorphism. Lithos, 2010, 114: 385-412

28 Li X P, Zheng Y F, Wu Y B, et al. Low-T eclogite in the Dabie terrane of China: Petrological and isotopic constraints on fluid activity and radiometric dating. Contrib Mineral Petrol, 2004, 148: 443-470

29 Zheng Y F, Gao X Y, Chen R X, et al. Zr-in-rutile thermometry of eclogite in the Dabieorogen: Constraints on rutile growth during continental subduction-zone metamorphism. J Asian Earth Sci, 2011, 40: 427-451

30 Gao X Y, Zheng Y F, Chen Y X. Dehydration melting of ultrahigh-pressure eclogite in the Dabieorogen: Evidence from multiphase solid inclusions in garnet. J Metamorph Geol, 2012, 30: 193-212

31 Ferrando S, Frezzotti M L, Dallai L, et al. Multiphase solid inclusions in UHP rocks (Su-Lu, China): Remnants of supercritical silicaterich aqueous fluids released during continental subduction. Chem Geol, 2005, 223: 68-81 